\title{
Slackening Effects in 2D Exact Positioning in Cable-Driven Parallel Manipulators
}

\author{
Erika Ottaviano ${ }^{1}$, Andrea Arena ${ }^{2}$, Vincenzo Gattulli ${ }^{2}$, and Francesco Potenza ${ }^{3}$ \\ 1 University of Cassino and Southern Lazio, Department of Civil and Mechanical \\ Engineering, Cassino, FR, 03043, Italy, ottaviano@unicas.it, \\ 2 Sapienza University of Rome, Department of Structural and Geotechnical \\ Engineering, Rome, 00184, Italy, andrea.arena@uniroma1.it, \\ vincenzo.gattulli@uniroma1.it, \\ 3 University of L'Aquila, Department of Civil Architectural and Environmental \\ Engineering, L'Aquila, 67100, Italy, francesco.potenza@univaq.it
}

\begin{abstract}
An analytical model of elastic cables is presented to evaluate the influence of the cables sag in the exact positioning of a point mass end-effector in a two-dimensional space within the context of cabledriven parallel manipulators. Direct and inverse kinematic problems are formulated by means of $3 n$ nonlinear equations expressing compatibility, equilibrium, and targeting conditions. Examples with an increasing number of cables are proposed to show that, in specific regions of the workspace, solving the inverse kinematics problem implies very slack configurations of redundant cables.
\end{abstract}

Keywords: cable-driven parallel robot, sag cables, inverse kinematics, direct kinematics

\section{Introduction}

Cable Driven Parallel Manipulators (CDPMs) have been developed since few decades and are currently studied because of their inherent advantages with respect their classical counterparts composed by rigid links only. The main element to consider in the study of the CDPMs is the cable and its correct modeling. The complexity is related to the use of cables as tools for actuation and manipulation of payloads. Indeed, cables have the characteristic of being only able to work in tension, and not in compression leading to many difficulties with kinematic, static, dynamic analyses and control. Some works are related to the low inertia of such mechanisms [7]. Applications taking advantage of the large working volume are the NIST Robocrane [4] and the systems proposed in [13, $2]$. When the CDPMs are in a crane configuration, where gravity acts like an additional cable, not all the end-effector DOFs can be controlled.

Underconstrained cable robots offer several advantages with respect to fully constrained ones. A smaller number of cables means reduced number of actuators, overall costs and setup time, improved ease of assembly and a lower likelihood of cable interference. Within the context of the underconstrained CDPMs 
kinematics, the challenging problem is that the exact positioning of the endeffector can be achieved with difficulty. Therefore, loop-closure or kinematic compatibility and equilibrium equations must be simultaneously solved as proposed in [14] and traditional displacement-analysis problem has been defined as geometrico-static also expressing some connections between stability and energy [3]. In [10] is solved the Inverse Kinematics (IK) problem considering the endeffector pose and evaluating the cables forces and lengths. The IK problem is solved in [8] with the hypothesis of inextensible cable first, and then using a parabolic cable profile equation. In [12] both Direct Kinematics (DC) and IK problems are solved within the context of sagged elastic cables. In [15] an approach based on energy and optimization was proposed to solve the DK problem. The energy method was also adopted in [5] to formulate the equations of motion of CDPMs to take into account cables vibrations. The importance of vibrations in cable-suspended robots and their mitigation via active control were discussed in [6]. When dealing with non-trivial cable models and underconstrained cable robots the main challenge is related to the real-time analysis capability of the controller. Indeed, simplified models allow fast computation but leading to inaccurate solutions, while non-trivial cable modeling may lead to ill-conditioned behavior at certain end-effector positions in the workspace.

In this paper an analytical model for solving numerically the DK and the IK problems in CDPMs working in a 2D space is presented. In particular, for a given, arbitrary number $n$ of elastic cables the solution of the IK and equilibrium problems is calculated starting from the solution of the DK approach and it is found in terms of the cables incremental lengths and tensions so as to provide the exact positioning of a point-mass end-effector across the workspace. To this aim, a kinematic descriptor, namely the vectorial distance between the endeffector position evaluated through a DK analysis and the target position, is minimized. It is also shown that there are specific regions in the $2 \mathrm{D}$ workspace where compatible and equilibrium solutions, ensuring the exact positioning of the end-effector, exist but entail very slack configurations of at least one cable.

\section{Problem Formulation}

In the two-dimensional (2D) Euclidean space, a system of $n$ elastic cables subjected to fixed boundary condition at one end and connected together at their free boundary is here considered to study the equilibrium configurations reached by the cables for assigned target positions of an end-effector point mass $M$ collocated at the cables free boundaries.

\subsection{Continuum Model of Elastic Cables}

The analytical model adopted to describe the cables overall 2D kinematics, illustrated in the schematic representation in Fig. 1, is parametrized by the space coordinate along the unstretched arclength $S_{i}(i=1, \ldots, n)$ of each cable. By introducing $n$ fixed Cartesian frames $\left(\mathbf{e}_{x}^{(i)}, \mathbf{e}_{y}^{(i)}\right)$ having their origin $O_{i}$ 
in the fixed boundary of the $i$ th cable, where $\mathbf{e}_{y}^{(i)}$ represents the upward vertical direction and $\mathbf{e}_{x}^{(i)}$ is collinear with the horizontal direction, the reference, unstretched configurations of the cables are described by the position vectors $\mathbf{p}_{i}\left(S_{i}\right)=x\left(S_{i}\right) \mathbf{e}_{x}^{(i)}+y\left(S_{i}\right) \mathbf{e}_{y}^{(i)}$, while the direction of the cables arclength is given by the unit vector $\mathbf{a}_{i}=a_{i, x} \mathbf{e}_{x}^{(i)}+a_{i, y} \mathbf{e}_{y}^{(i)}$. On the other hand, the unstretched cables configuration is assigned a priori by assuming a trial collocation of the mass $M$ whose position, in the frame $\left(\mathbf{e}_{x}^{(1)}, \mathbf{e}_{y}^{(1)}\right)$ of the first cable, is given by the position vector $\mathbf{p}_{M, 1}^{0}=l_{1} \mathbf{a}_{1}^{0}$, where $l_{1}$ and $\mathbf{a}_{1}^{0}=a_{1, x}^{0} \mathbf{e}_{x}^{(1)}+a_{1, y}^{0} \mathbf{e}_{y}^{(1)}$ are the distance and the direction, respectively, between the cable fixed end and the mass $M$. Accordingly, the position of the fixed end of the $i$ th cable is known and it is assigned through the vector $\mathbf{r}_{O_{i}}=d_{1 i} \mathbf{e}_{x}^{(1)}+h_{1 i} \mathbf{e}_{y}^{(1)}(i=1, \ldots, n)$, where $d_{1 i}$ and $h_{1 i}$ are the horizontal and vertical distances, respectively, between the fixed ends of the first and the $i$ th cable. The remaining $n-1$ expressions of the mass position vectors $\mathbf{p}_{M, i}^{0}$ with respect to the $i$ th frame, can be determined by the $n-1$ vectorial relations $\mathbf{r}_{O_{i}}+\mathbf{p}_{M, i}^{0}=\mathbf{p}_{M, 1}^{0}$; the latter, can be written in component form as $d_{1 i}+l_{i} a_{i, x}^{0}=l_{1} a_{1, x}^{0}, h_{1 i}+l_{i} a_{i, y}^{0}=l_{1} a_{1, y}^{0}$, which, together with the normalization condition $\left(a_{i, x}^{0}\right)^{2}+\left(a_{i, y}^{0}\right)^{2}=1$, provides the expressions of $l_{i}, a_{i, x}^{0}$, and $a_{i, y}^{0}(i=2, \ldots, n)$, respectively.

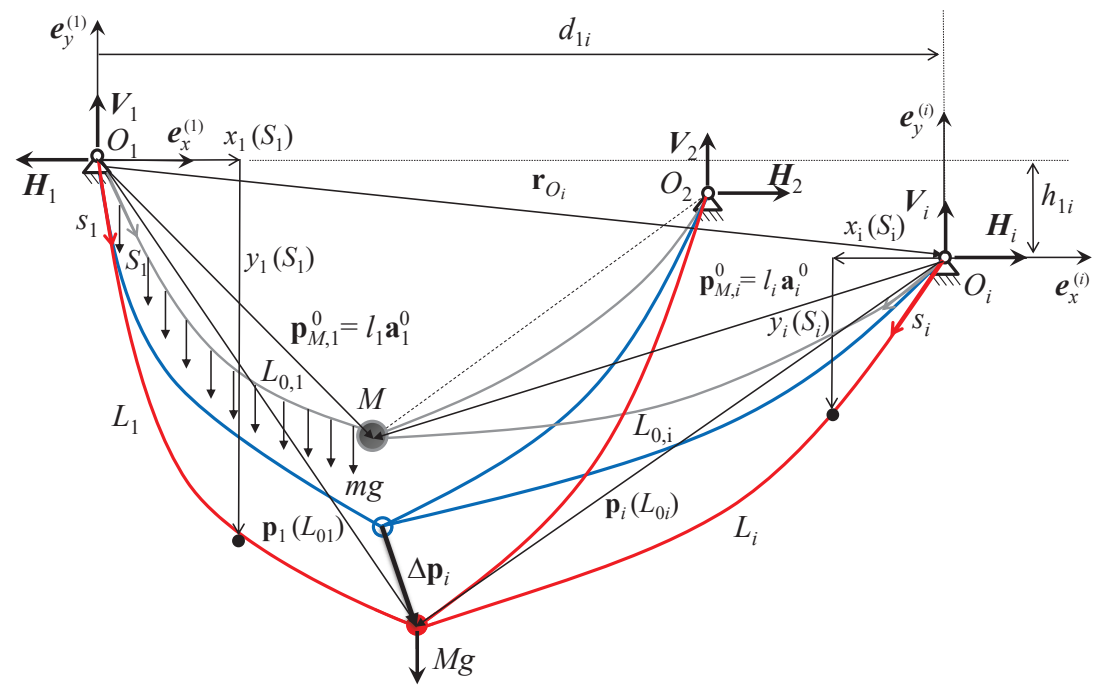

Fig. 1. Schematic representation of the CDPM.

In the continuum formulation here adopted, the flexural deformation mode of the cables [1] is neglected, thus the only generalized strain of the $i$ th cable is provided by the stretch $\nu_{i}$. The latter, can be defined as $\nu_{i}=d s_{i} / d S_{i}$, where $s_{i}$ represents the arclength of the $i$ th cable in the equilibrium configuration and $d$ 
stands for total differentiation. The stress state in the $i$ th cable is given by the vector $\mathbf{n}_{i}=N_{i} \mathbf{a}_{i}$, where, by considering a linear elastic constitutive behavior, the tension $N_{i}$ can be expressed in terms of the cable stretch as $N_{i}=E A_{i}\left(\nu_{i}-1\right)$, being $E A_{i}$ the cable axial stiffness.

By introducing the horizontal $H_{i}$ and the vertical $V_{i}$ components of the cable tension in the $i$ th reference frame, the following equilibrium equations for the $i$ th cable holds

$$
\begin{aligned}
& N_{i} a_{i, x}=H_{i}, \\
& N_{i} a_{i, y}=-V_{i}+\int_{0}^{S_{i}} m g d S_{i} .
\end{aligned}
$$

where the integral term in the right-hand side of the second equation in (1) represents the weight of the $i$ th cable having unstretched length $S_{i}$, i.e., $m g S_{i}=$ $\int_{0}^{S_{i}} m g d S_{i}$, being $m$ the cable mass per unit unstretched length and $g$ the gravity acceleration. By solving Eq.(1) in terms of the directors $a_{i, x}$ and $a_{i, y}$, and providing the normalization condition $a_{i, x}^{2}+a_{i, y}^{2}=1$, it is possible to obtain th expression of the tension in the $i$ th cable in the form $N_{i}=\sqrt{S_{i}^{2}+H_{i}^{2}+V_{i}^{2}-2 S_{i} V_{i}}$.

By now enforcing the definition of the stretch $\nu_{i}$, which implies that $d s_{i}=$ $\nu_{i} d S_{i}$, the directors of the $i$ th unit vector $\mathbf{a}_{i}$ can be cast in the following form: $a_{i, x}=d x_{i} / d s_{i}=d x_{i} / \nu_{i} d S_{i}, a_{i, y}=d y_{i} / d s_{i}=d y_{i} / \nu_{i} d S_{i}$. By combining the obtained expressions of the directors $a_{i, x}$ and $a_{i, y}$ and the constitutive equation is then possible to obtain the differential relations between the components of the position vector $\mathbf{p}_{i}\left(S_{i}\right)$ of the $i$ th cable and the unstretched arclength $S_{i}$ in the form

$$
d x_{i}=a_{i, x}\left(\frac{N_{i}}{E A_{i}}+1\right) d S_{i}, d y_{i}=a_{i, y}\left(\frac{N_{i}}{E A_{i}}+1\right) d S_{i}
$$

with: $a_{i, x}=H_{i} / N_{i}$ and $a_{i, y}=\left(m g S_{i}-V_{i}\right) / N_{i}$. Equation (2), integrated in $\left[0, S_{i}\right]$, provides the equilibrium configuration of the $i$ th cable in terms of the horizontal and the vertical components of the position vector $\mathbf{p}_{i}\left(S_{i}\right)$.

Nondimensional form. The nondimensional form of Eq.(2) and of the equilibrium equations (1) are obtained here to identify the main mechanical parameters governing the static configuration of the $i$ th elastic cable. To this end, the span $l_{1}$ of the first cable is adopted as characteristic geometric parameter for the nondimensionalization. Thus, $\xi_{i}=S_{i} / l_{1}$ is the nondimensional arclength in the unstretched configuration, while $\bar{x}_{i}=x_{i} / l_{1}$ and $\bar{y}_{i}=y_{i} / l_{1}$ represent the horizontal and vertical components of the $i$ th cable position vector in the equilibrium configuration, respectively. Moreover, by assuming $f^{c}=m g l_{1}$ as characteristic force, the following nondimensional expressions of the components of the cables tension can be provided: $\alpha_{i}=H_{i} / m g l_{1}, \beta_{i}=V_{i} / m g l_{1}$. Finally, the nondimensional tension in the $i$ th cable can be written as $\bar{N}_{i}=N_{i} / m g l_{1}=$ $\kappa_{i}\left(\nu_{i}-1\right)$, where $\kappa_{i}=E A_{i} / m g l_{1}$ is the nondimensional cable stiffness, thus $\bar{N}_{i}=\sqrt{\xi_{i}^{2}+\alpha_{i}^{2}+\beta_{i}^{2}-2 \xi_{i} \beta_{i}}$. By now integrating Eq.(2), the equilibrium config- 
uration of the $i$ th cable, in its nondimensional form, can be calculated as [9]

$$
\begin{aligned}
& \bar{x}_{i}\left(\xi_{i}\right)=\bar{x}_{i}(0)+\alpha_{i}\left[\frac{\xi_{i}}{\kappa_{i}}+\sinh ^{-1}\left(\frac{\xi_{i}-\beta_{i}}{\alpha_{i}}\right)+\sinh ^{-1}\left(\frac{\beta_{i}}{\alpha_{i}}\right)\right], \\
& \bar{y}_{i}\left(\xi_{i}\right)=\bar{y}_{i}(0)+\frac{\xi_{i}\left(\xi_{i}-2 \beta_{i}\right)}{2 \kappa_{i}}+\sqrt{\xi_{i}^{2}+\alpha_{i}^{2}+\beta_{i}^{2}-2 \xi_{i} \beta_{i}}-\sqrt{\alpha_{i}^{2}+\beta_{i}^{2}},
\end{aligned}
$$

where $\sinh ^{-1}$ is the inverse hyperbolic sine function. Finally, to ensure the fixed boundary conditions at the cables ends, $\bar{x}_{i}(0)=\bar{y}_{i}(0)=0(i=1, \ldots, n)$.

\subsection{Equilibrium and Kinematically Compatible Configurations}

The system (3) provides the nondimensional expression of the configuration of the $i$ th cable as function of the elastic stiffness $\kappa_{i}$ and of the internal forces $\alpha_{i}$ and $\beta_{i}$. Although, to ensure that the $n$ cables are in equilibrium under the effect of the external forces acting on them, the following two relations hold

$$
\sum_{i=1}^{n} \alpha_{i}=0, \sum_{i=1}^{n} \beta_{i}-\sum_{i=1}^{n} \eta_{i}-\bar{W}=0,
$$

where $\bar{W}=M g / m g l_{1}$ is the nondimensional weight of the point mass $M$, while $\eta_{i}=m g L_{0, i} / m g l_{1}$ represents the nondimensional weight of the $i$ th cable, which, in turn, corresponds to the ratio between the unstretched length $L_{0, i}$ of the $i$ th cable and the span $l_{1}$ of the first cable, i.e., $\eta_{i}=L_{0, i} / l_{1}$.

In addition, kinematic compatibility conditions must be provided in order to ensure that the free boundaries of the $n$ cables are connected to each other. To this aim, the following $n-1$ vectorial relations hold

$$
\overline{\mathbf{r}}_{O_{i}}+\overline{\mathbf{p}}_{i}\left(\eta_{i}\right)-\overline{\mathbf{p}}_{1}\left(\eta_{1}\right)=\mathbf{o},(i=2, \ldots, n)
$$

where $\overline{\mathbf{r}}_{O_{i}}=\mathbf{r}_{O_{i}} / l_{1}, \overline{\mathbf{p}}_{i}\left(\eta_{i}\right)$ and $\overline{\mathbf{p}}_{1}\left(\eta_{1}\right)$ are the nondimensional position vectors of the mass $M$ with respect to the fixed end of the $i$ th cable (i.e., when $\xi_{i}=\eta_{i}$ ) and the first cable (i.e., when $\xi_{1}=\eta_{1}$ ), respectively, and $\mathbf{o}$ is the trivial vector with null components.

\section{Direct vs Inverse Approach}

Equation (5), in component form, provides $2(n-1)$ compatibility conditions which, together with the 2 equilibrium equations (4), give the $2 n$ equations that allow to calculate the $2 n$ unknowns $\alpha_{i}$ and $\beta_{i}(i=1, \ldots, n)$ ensuring that the mechanical system is kinematically compatible and in equilibrium. The above mentioned system of $2 n$ nonlinear equations in $2 n$ unknowns can be solved numerically for selected values of the cables unstretched lengths $L_{0, i}$, assigned through the nondimensional parameters $\eta_{i}$, and for a set of trial positions of the mass $M$, which, in turn, are provided by the nondimensional position vectors $\overline{\mathbf{p}}_{M, i}^{0}=\mathbf{p}_{M, i}^{0} / l_{1}$. Although, due to the elastic feature of the cables, the final position of the mass (given in the $i$ th reference frame by the vector $\overline{\mathbf{p}}_{i}\left(\eta_{i}\right)$ ) will not be coincident to the assigned trial position. 
Finding a closed-form solution of the system of the $2 n$ nonlinear equations is challenging and out of the scope of the present work; nevertheless, approximate solutions can be found numerically by using minimization methods. To this end, a numerical algorithm for constrained global optimization implemented in the software Mathematica was used. In particular, the Random Search algorithm accounting for a large number of search points is adopted to minimize the objective function given by the squared norm of the $(2 n \times 1)$ vector collecting the left-hand side $(L H S)$ of the system of $2 n$ nonlinear equations. To overcome the limits of the direct kinematic approach in finding the cables configurations leading to the mass exact positioning, an inverse kinematic approach is adopted to drive the position of the end-effector to target collocations. To this end, the target position vector $\overline{\mathbf{p}}_{T}$, whose components are given in the fixed frame $\left(O_{1}, \mathbf{e}_{x}^{(1)}, \mathbf{e}_{y}^{(1)}\right)$ of the first cable, is introduced to define the difference vector between the position of the mass evaluated through a direct kinematic analysis and the target position vector. Since the mass position, in the stretched cables configurations, can be arbitrary provided with respect to each cable by the sum vector $\overline{\mathbf{r}}_{O_{i}}+\overline{\mathbf{p}}_{i}\left(\eta_{i}\right)$, therefore, it is possible to evaluate $n$ difference vectors $\Delta \overline{\mathbf{p}}_{i}(i=1, \ldots, n)$ as $\Delta \overline{\mathbf{p}}_{i}=\overline{\mathbf{r}}_{O_{i}}+\overline{\mathbf{p}}_{i}\left(\eta_{i}\right)-\overline{\mathbf{p}}_{T}$; the latter turn out to be $\Delta \overline{\mathbf{p}}_{i} \neq \mathbf{o}$ in the configurations achieved through a direct kinematic approach. On the other hand, the mass target position is reached when $\Delta \overline{\mathbf{p}}_{i}=\mathbf{o}$.

Starting from the known configurations attained through the DK, equilibrium equations (4) and compatibility equations (5) can be written in their incremental form by introducing the following expressions: $\alpha_{i}=\alpha_{i}^{0}+\Delta \alpha_{i}, \beta_{i}=\beta_{i}^{0}+\Delta \beta_{i}$ and $\eta_{i}=\eta_{i}^{0}+\Delta \eta_{i}$, where $\alpha^{0}$ and $\beta_{i}^{0}$ are solution of the direct problem, while $\eta_{i}^{0}$ are the assigned parameters providing the unstretched cables lengths. Therefore, $3 n$ incremental unknowns, namely $\Delta \alpha_{i}, \Delta \beta_{i}$, and $\Delta \eta_{i}(i=1, \ldots, n)$, are introduced and the solution of the inverse kinematic problem can be then numerically searched by simultaneously solving the set of $3 n$ equations given by

$$
\begin{aligned}
& \sum_{i=1}^{n}\left(\alpha_{i}^{0}+\Delta \alpha_{i}\right)=0, \\
& \sum_{i=1}^{n}\left(\beta_{i}^{0}+\Delta \beta_{i}\right)-\sum_{i=1}^{n}\left(\eta_{i}^{0}+\Delta \eta_{i}\right)-\bar{W}=0, \\
& \overline{\mathbf{r}}_{O_{i}}+\overline{\mathbf{p}}_{i}\left(\eta_{i}^{0}+\Delta \eta_{i}\right)-\overline{\mathbf{p}}_{1}\left(\eta_{1}^{0}+\Delta \eta_{1}\right)=\mathbf{o},(i=2, \ldots, n), \\
& \left\|\Delta \overline{\mathbf{p}}_{i}\right\|=0,(i=1, \ldots, n),
\end{aligned}
$$

where the last of (6) represents $n$ target equations. In accordance with the numerical procedure performed to find the approximate solution of the direct problem, which depends largely on a good initial guess [12], the numerical global optimization based on the Random Search algorithm is adopted to minimize the objective function given, for the case of the inverse kinematics, by the squared norm of the $(3 n \times 1)$ vector collecting the left-hand side $(L H S)$ of the nonlinear equations system (6). 


\subsection{Mechanical Parameters of the Selected CDPMs}

Two cables configurations are investigated, namely, case-study A (CSA) and case-study B (CSB), respectively. In particular, CSA accounts for three suspended cables having their fixed end at the same height and being symmetrically positioned along the horizontal direction, while in CSB are considered four cables nonsymmetrically positioned in the workspace. By referring to the mechanical parameters reported in [14], in both cases the cables are characterized by the same axial stiffness $E A_{i}=628.319 \mathrm{~N}(i=1, \ldots, n)$ and the end-effector mass is $M=5.326 \times 10^{-2} \mathrm{~kg}$. Moreover, the positions of the cables fixed end are assumed as follows: in CSA, $d_{12}=1 \mathrm{~m}, d_{13}=2 \mathrm{~m}$ and $h_{12}=0=h_{13}$, while in CSB, $d_{12}=0.5 \mathrm{~m}, d_{13}=1.25 \mathrm{~m}, d_{14}=2 \mathrm{~m}$, and $h_{12}=-0.1 \mathrm{~m}, h_{13}=0.25$ $\mathrm{m}, h_{14}=0.15 \mathrm{~m}$.
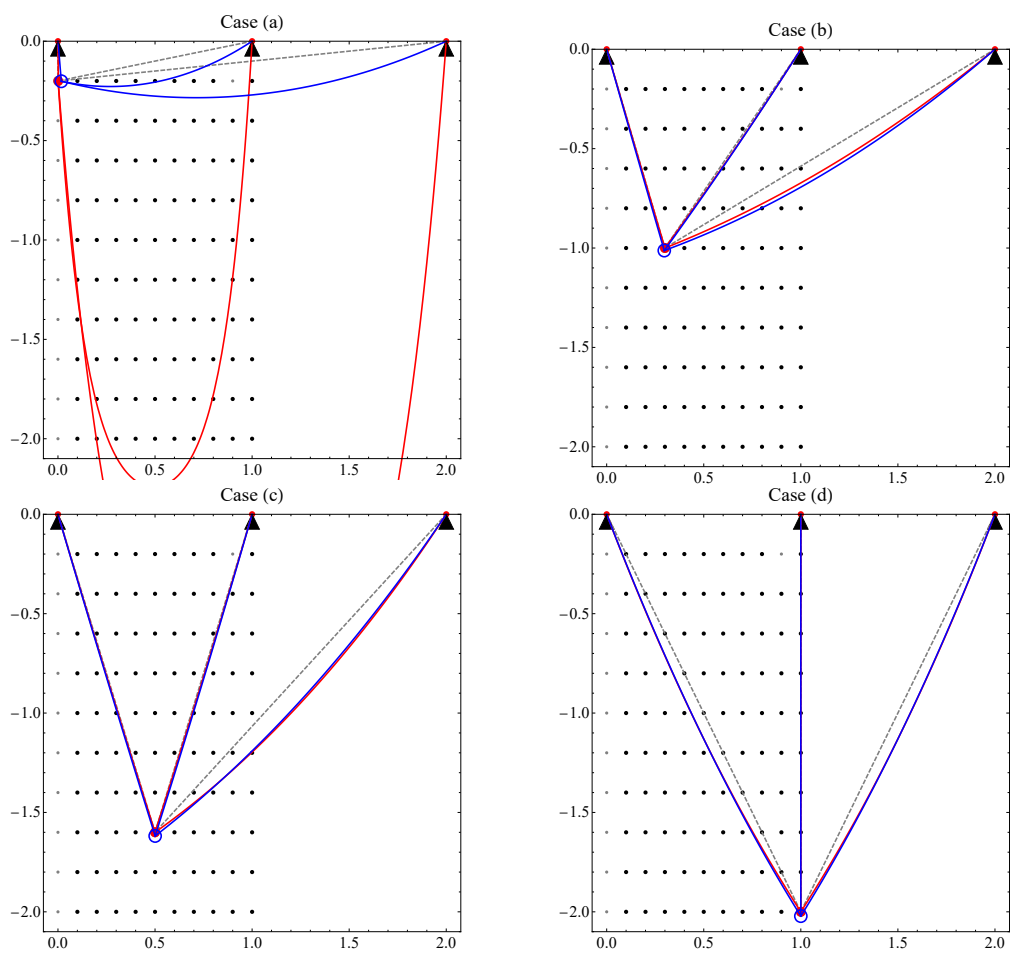

Fig. 2. Cables equilibrium configurations $\left(\Lambda_{i}^{0}=1.01\right)$ for CSA: direct approach (blue lines) vs. inverse approach (red lines). The gray dashed lines indicate the distances $l_{i}$ $(i=1, \ldots, n)$; dimensions in $[\mathrm{m}]$.

The reference configuration of the $i$ th cable is provided by assigning the cable aspect ratio $\Lambda_{i}^{0}[9,11]$, that is, the ratio between the unstretched cable length $L_{0, i}$ and the distance $l_{i}$ between its fixed end and the mass target position. Conse- 
quently, the nondimensional unstretched length of the $i$ th cable can be calculated as $\eta_{i}^{0}=\Lambda_{i}^{0} l_{i} / l_{1}$. As demonstrated in [14], the sensitivity of the end-effector mass positioning with respect to the prescribed parameter $\Lambda_{i}^{0}$ is a study of valuable interest in the case of direct kinematic approach, where the accurate tuning of $\Lambda_{i}^{0}$ may provide an end-effector mass positioning close, although not coincident, to the target position. On the other hand, in the inverse kinematic approach, the final cables aspect ratios are provided by the minimization procedure and can be calculated as $\Lambda_{i}=\left(\eta_{i}^{0}+\Delta \eta_{i}\right) l_{1} / l_{i}$. Therefore, in both case-studies here investigated, $\Lambda_{i}^{0}=1.01$ corresponds to the initial, tentative, aspect ratio for each cable (i.e., $i=1, \ldots, n)$.

Table 1. CSA: coordinates (in [m]) of the target points compared with the end-effector mass position reached through the direct and the inverse approach, respectively. $\mathrm{Nu}-$ merical approximation of the LHS of Eq. (6).

\begin{tabular}{l|cc|cc|cc|cc}
\hline Case & $x_{T}$ & $y_{T}$ & $x_{D}$ & $y_{D}$ & $x_{I}$ & $y_{I}$ & $\|L H S\|^{2}$ & $\|L H S\|$ \\
\hline (a) & 0 & -0.2 & 0.0172 & -0.2014 & 0.00169 & -0.1999 & $1.43 \times 10^{-4}$ & $1.19 \times 10^{-2}$ \\
\hline (b) & 0.3 & -1 & 0.2966 & -1.0126 & 0.3 & -1 & $8.09 \times 10^{-29}$ & $8.99 \times 10^{-15}$ \\
(c) & 0.5 & -1.6 & 0.5006 & -1.6183 & 0.5 & -1.6 & $2.57 \times 10^{-29}$ & $5.07 \times 10^{-15}$ \\
(d) & 1 & -2 & 1 & -2.0214 & 1 & -2 & $1.68 \times 10^{-21}$ & $4.09 \times 10^{-11}$ \\
\hline
\end{tabular}

In Fig. 2 are shown, for the CSA, the equilibrium configurations undergone by the cables for 4 selected end-effector positions across the grid of target points investigated. Accordingly, Tab. 1 shows for the cases displayed in Fig. 2 the final positions of the end-effector mass attained through the direct approach (i.e., $x_{D}$ and $y_{D}$ ) and through the inverse approach (i.e., $x_{I}$ and $y_{I}$ ) compared with the selected target positions (i.e., $x_{T}$ and $y_{T}$ ). The last two columns of Tab. 1 report the approximation of the numerical solution of the nonlinear system (6) in terms of the squared norm $\|L H S\|^{2}$ and of the norm $\|L H S\|$ of its leftend side expressions. Due to the superimposed value of $\Lambda_{i}^{0}=1.01$, the cables configurations attained by the direct kinematics approach (blue lines in Fig. 2) are non-slack in the whole workspace, although, the final end-effector position does not match the target points due to the effect of the cables elasticity, as shown in Tab. 1. On the other hand, the formulation based on the inverse kinematics (red lines in Fig. 2) allows to obtain the cables elastic configurations such as to provide the exact mass positioning (see $x_{I}$ and $y_{I}$ in Tab. 1). Nevertheless, as depicted in Fig. 2 (Case (a)), several target points cannot be reached by ensuring non-slack $\Lambda_{i} \leq 1$ or moderately slack configurations for all the cables. This results in a higher residual of the LHS of Eq. (6) and in larger cables aspect ratios $\Lambda_{i}>>1$, as shown in Tab. 2 where, together with the values of $\Lambda_{i}$, are reported the cables axial forces $N_{i}$ in the proximity of the mass and the cables stretched lengths $L_{i}$. Finally, in Fig. 3, are reported the axial forces in the proximity of the end-effector and the cables total length for all target points reached through non-slack or moderately slack configurations. 
Table 2. CSA: results in terms of axial forces $N_{i}$ (in [N]), cables total stretched lengths $L_{i}$ (in $[\mathrm{m}]$ ), and cables aspect ratio $\Lambda_{i}$.

\begin{tabular}{l|ccc|ccc|ccc}
\hline Case & $N_{1}$ & $N_{2}$ & $N_{3}$ & $L_{1}$ & $L_{2}$ & $L_{3}$ & $\Lambda_{1}$ & $\Lambda_{2}$ & $\Lambda_{3}$ \\
\hline (a) & 0.588 & 0.024 & 0.042 & 0.199 & 4.565 & 7.854 & 0.999 & 4.48 & 3.91 \\
\hline (b) & 0.425 & 0.119 & 0.057 & 1.044 & 1.221 & 1.980 & 0.999 & 0.999 & 1.004 \\
(c) & 0.314 & 0.213 & 0.037 & 1.676 & 1.676 & 2.206 & 0.999 & 0.999 & 1.006 \\
(d) & 0.043 & 0.451 & 0.043 & 2.240 & 2.000 & 2.240 & 1.002 & 0.999 & 1.002 \\
\hline
\end{tabular}
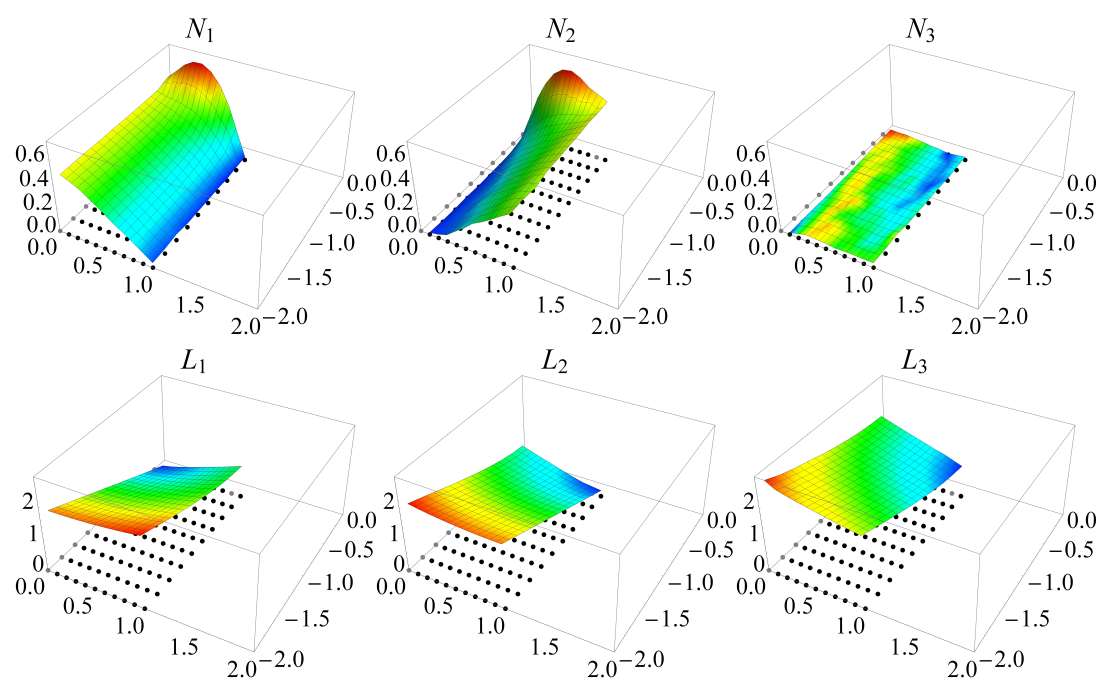

Fig. 3. Axial forces $N_{i}$ (in $[\mathrm{N}]$ ) and total lengths $L_{i}$ (in $[\mathrm{m}]$ ) for each target position of CSA (coordinates are in $[\mathrm{m}])$.

To show the capability of the proposed model to deal with generic cables arrangements, the case of four, nonsymmetrically positioned cables (i.e., the CSB) is investigated next. Figure 4 shows the cables configurations for selected the target points; in particular, case (a) shows very slack configurations attained in a selected target point positioned along the boundary of the workspace (i.e., gray points at $x=0 \mathrm{~m}$ ) by the three cables positioned far away from the boundary, while cases (b), (c) and (d) show the non-slack and the moderately slack configurations undergone by all cables to reach the target points inside the workspace. Blue and red lines represent the configurations obtained via the DK and the IK approach, respectively. In Tab. 3 and Tab. 4 are reported the numerical errors and the cables mechanical parameters for the selected four cases of CSB. In Fig. 5 are shown, for CSB, the axial forces in correspondence with the end-effector and the cables total length for all target points reached through non-slack or moderately slack configurations. 
Table 3. CSB: coordinates (in $[\mathrm{m}]$ ) of the target points compared with the end-effector mass position reached through the direct and the inverse approach, respectively. Numerical approximation of the $L H S$ of Eq. (6).

\begin{tabular}{l|cc|cc|cc|cc}
\hline Case & $x_{T}$ & $y_{T}$ & $x_{D}$ & $y_{D}$ & $x_{I}$ & $y_{I}$ & $\|L H S\|^{2}$ & $\|L H S\|$ \\
\hline (a) & 0 & -0.5 & 0.0396 & -0.5039 & 0.0034 & -0.4999 & $1.1 \times 10^{-4}$ & $1.1 \times 10^{-2}$ \\
\hline (b) & 0.25 & -0.975 & 0.2525 & -0.9854 & 0.25 & -0.975 & $5.9 \times 10^{-28}$ & $2.4 \times 10^{-14}$ \\
(c) & 0.5 & -1.2125 & 0.5056 & -1.2244 & 0.5 & -1.2125 & $2.7 \times 10^{-15}$ & $5.2 \times 10^{-8}$ \\
\hline (d) & 1.75 & -1.925 & 1.7387 & -1.9463 & 1.75 & -1.925 & $1.6 \times 10^{-27}$ & $4.1 \times 10^{-14}$ \\
\hline
\end{tabular}
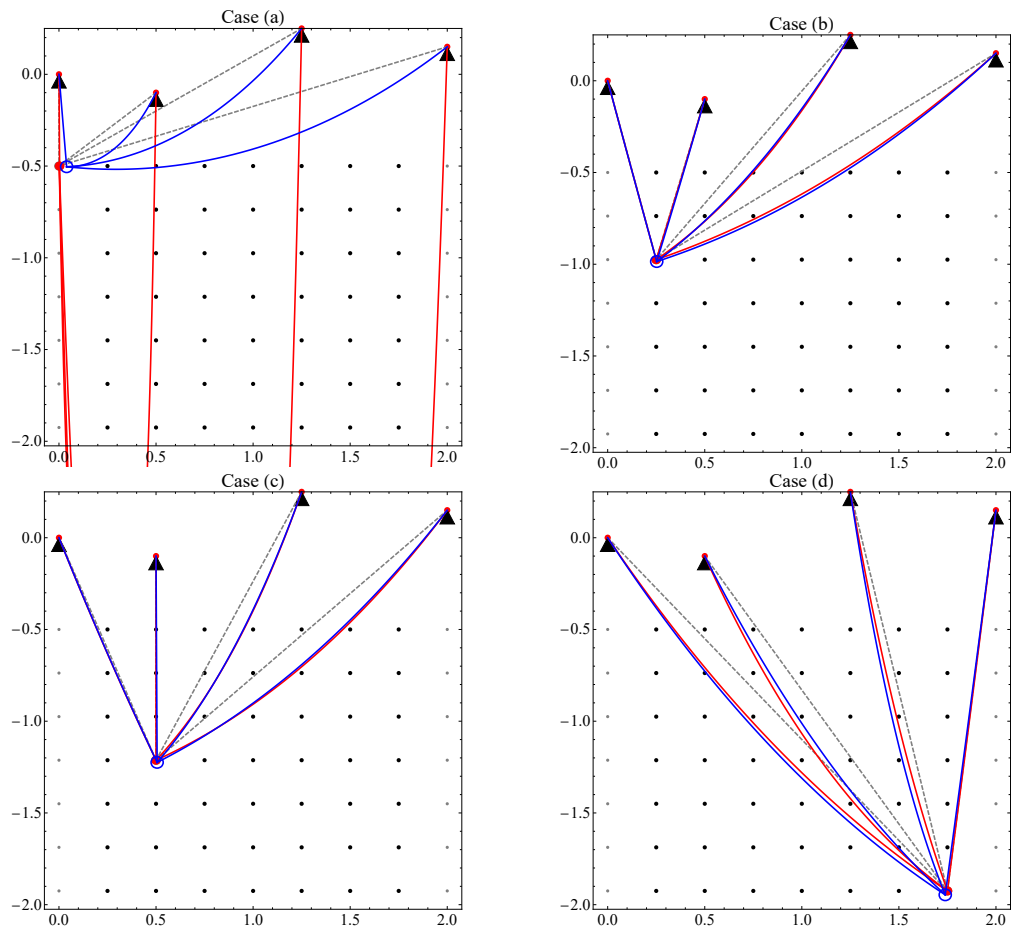

Fig. 4. Cables equilibrium configurations $\left(\Lambda_{i}^{0}=1.01\right)$ for CSB: direct approach (blue lines) vs. inverse approach (red lines). The gray dashed lines provide the distances $l_{i}$ $(i=1, \ldots, n)$; dimensions are in $[\mathrm{m}]$.

A deeper investigation of the cables configurations in the neighborhood of the target points positioned along the workspace boundaries (i.e., $x_{T}=0 \mathrm{~m}$ and $x_{T}=2 \mathrm{~m}$ ) was performed next to define the workspace of the CDPM where the cables are non-slack or moderately slack (i.e., $\Lambda_{i} \approx 1$ ). For both case-studies (i.e., CSA and CSB) a thicker grid of target points was used to determine, via the inverse kinematic approach, the end-effector positions where all cables exhibit a moderately slack configuration, i.e., $\Lambda_{i}<1$.1. In Fig. 6 are shown in red 
Table 4. CSB: results in terms of axial forces $N_{i}$ (in [N]), cables total stretched lengths $L_{i}$ (in $[\mathrm{m}]$ ), and cables aspect ratio $\Lambda_{i}$.

\begin{tabular}{l|cccc|cccc|cccc}
\hline Case & $N_{1}$ & $N_{2}$ & $N_{3}$ & $N_{4}$ & $L_{1}$ & $L_{2}$ & $L_{3}$ & $L_{4}$ & $\Lambda_{1}$ & $\Lambda_{2}$ & $\Lambda_{3}$ & $\Lambda_{4}$ \\
\hline (a) & 0.703 & 0.034 & 0.066 & 0.0799 & 0.499 & 6.634 & 12.78 & 15.2 & 0.999 & 10.36 & 8.767 & 7.226 \\
\hline (b) & 0.376 & 0.139 & 0.019 & 0.042 & 1.007 & 0.910 & 1.594 & 2.095 & 0.9994 & 0.9998 & 1.0079 & 1.0071 \\
(c) & 0.083 & 0.424 & 0.015 & 0.027 & 1.312 & 1.112 & 1.654 & 2.048 & 1.0 & 0.9993 & 1.0063 & 1.0106 \\
\hline (d) & 0.041 & 0.020 & 0.025 & 0.467 & 2.618 & 2.235 & 2.234 & 2.090 & 1.0062 & 1.0104 & 1.0009 & 0.9992 \\
\hline
\end{tabular}
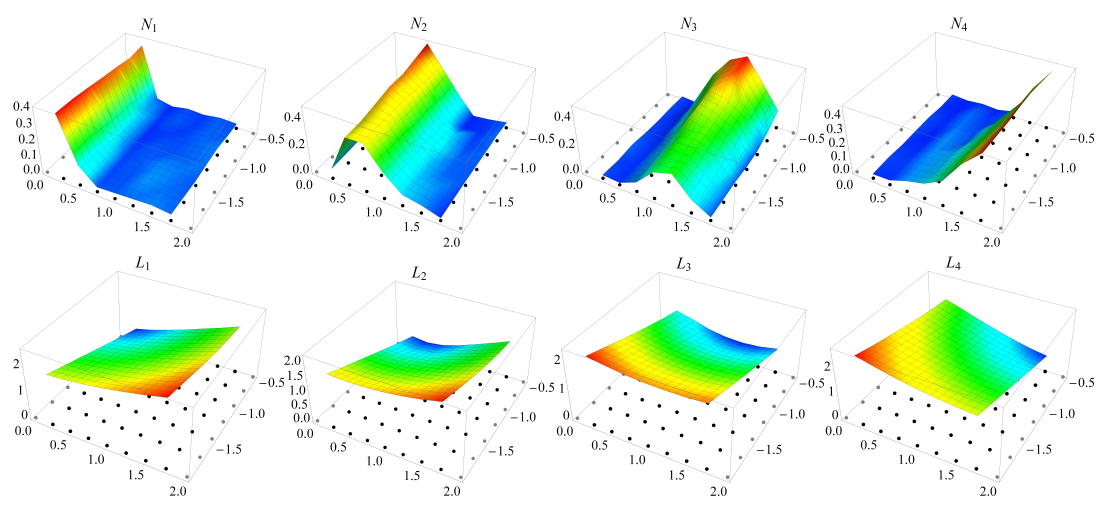

Fig. 5. Axial forces $N_{i}$ (in $[\mathrm{N}]$ ) and total lengths $L_{i}$ (in $[\mathrm{m}]$ ) for each target position of CSB (coordinates are in $[\mathrm{m}]$ ).

the target points for which at least one cable results very slack (i.e. $\Lambda>1.1$ ), therefore redundant, while black points indicate the target positions reached by non-slack or moderately slack cables configurations.
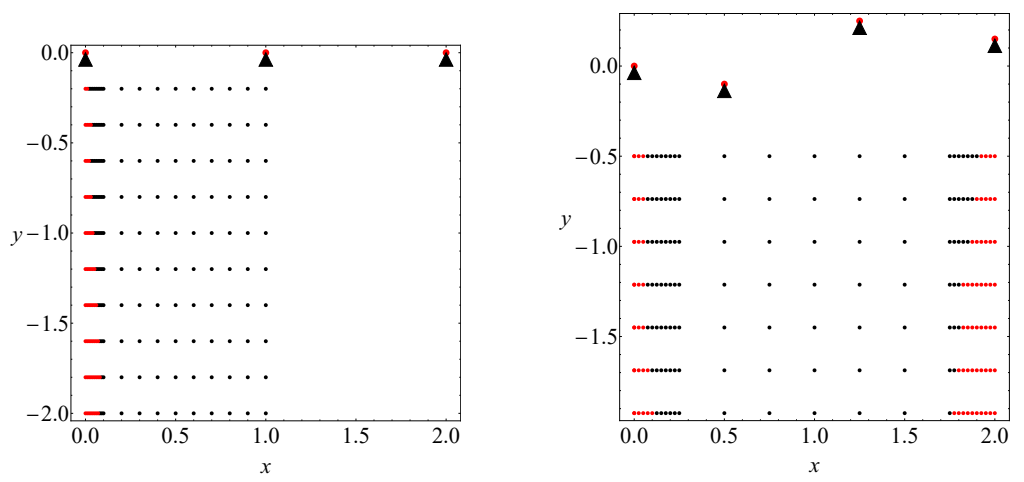

Fig. 6. Target points for CSA (left) and CSB (right) where at least one cable possesses a very slack configuration (red) and where all cables are non-slack or moderately slack (black); coordinates are in $[\mathrm{m}]$. 


\section{Conclusions}

The studies performed in this paper show that the exact positioning of the end-effector in specific area of the workspace of CDPMs may imply a large sag in "redundant" cables. This occurrence is due to the small contribute of these cables to the equilibrium of the end-effector and cannot be studied with simplified models of CDPMs. The presented model permits to determine reachable portion of the workspace when the maximum cable sag is given.

\section{Acknowledgements}

This work was partially supported by EU Call RFCS-2017 through the research project DESDEMONA (grant agreement number 800687).

\section{References}

1. Arena, A., Pacitti, A., Lacarbonara W.: Nonlinear response of elastic cables with flexural-torsional stiffness. Int J Solids Struct, 87, 267-277 (2016).

2. Bosscher, P.: Cable-suspended robotic contour crafting system. Automat Constr 17: 45-55 (2006).

3. Carricato, M., Merlet, J-P.: Stability analysis of underconstrained cable-driven parallel robots. IEEE Trans. on Robotics 29(1):288-296 (2013).

4. Castelli, G., Ottaviano, E., Rea, P.: A Cartesian Cable-Suspended Robot for improving end-users' mobility in an urban environment. Robot Cim-Int Manuf 30(3): 335-343 (2014).

5. Du, J., Agrawal, SK.: Dynamic Modeling of Cable-Driven Parallel Manipulators with Distributed Mass Flexible Cables. J Vib Acoust 137(2): 1-8 (2015).

6. Gattulli, V., Alaggio, R., Potenza, F.: Analytical Prediction and Experimental Validation for Longitudinal Control of Cable Oscillations. Int J Nonlin Mech 43: 36-52 (2008).

7. Gonzalez-Rodriguez, A., Castillo-Garcia, F.J., Ottaviano, E., Rea, P., GonzalezRodriguez, A.G.:On the effects of the design of cable-Driven robots on kinematics and dynamics models accuracy. Mechatronics 43: 18-27 (2017).

8. Gouttefarde, M., Collard, J., Riehl, N., Baradat, C.: Simplified static analysis of large-dimension parallel cable-driven robots. IEEE International Conference on Robotics and Automation, Saint Paul, MN: 2299-2305 (2012).

9. Irvine, H.: Cable Structures. Cambridge, MA: MIT Press (1981).

10. Kozak et al.: Static Analysis of Cable-Driven Manipulators with Non-Negligible Cable Mass. IEEE Trans. on Robotics 22(3):425-433 (2006).

11. Lepidi, M., Gattulli, V.: Static and Dynamic Response of Elastic Suspended Cables with Thermal Effects. Int J Solids Struct 49(9): 1103-1116 (2012).

12. Merlet, J.-P., dit Sandretto, A.: The Forward Kinematics of Cable-Driven Parallel Robots with Sagging Cables. In Cable-Driven Parallel Robots: 3-15 (2014) Springer.

13. Nan, R., Peng, B.: A chinese concept for $1 \mathrm{~km}$ radio telescope. Acta Astronaut 46(10-12): 667-675 (2000).

14. Ottaviano, E., Gattulli, V., Potenza, F.: Elasto-Static Model for Point Mass Sagged Cable-Suspended Robots. Advances in Robot Kinematics 4:351-359 (2018).

15. Pott, A, Tempel, P.: A Unified Approach to Forward Kinematics for Cable-Driven Parallel Robots Based on Energy. Advances in Robot Kinematics 8:401-409 (2019). 